\title{
Influência de Adjuvantes e Pontas de Pulverização na Deriva DE APLicaÇÃo do Glyphosate ${ }^{1}$
}

\author{
Influence of Adjuvants and Spray Nozzles on Glyphosate Drift
}

\author{
MARTINI, A.T. ${ }^{2}$, AVILA, L.A. ${ }^{2}$, CAMARGO, E.R. ${ }^{2}$, MOURA, D.S. ${ }^{2}$, MARCHEZAN, M.G. ${ }^{2}$ e \\ PIVETTA, A.P. ${ }^{2}$
}

\begin{abstract}
RESUMO - Além do impacto ambiental, a deriva de glyphosate pode causar danos sobre culturas sensiveis a esse herbicida. O objetivo deste estudo foi avaliar a influência de adjuvantes e pontas de pulverização na deriva de glyphosate aplicado via terrestre. O estudo foi conduzido em campo, arranjado em esquema fatorial ( $3 \times 9)$, sendo o primeiro fator constituído por três pontas de pulverização: uma de jato plano (Teejet XR 110.015) e duas de jato plano com indução de ar (Teejet AIXR 110.015 e Teejet TTI 110.015). O segundo fator foi composto por sete adjuvantes: Break Thru ${ }^{\circledR}\left(0,1 \%\right.$ v.v.), Grap' Oil ${ }^{\circledR}(0,5 \%$ v.v.), Grap Super $\operatorname{Gun}^{\circledR}\left(0,03 \%\right.$ v.v.), Iharol ${ }^{\circledR}(1 \%$ v.v. $)$, NP- $10^{\circledR}\left(0,075\right.$ L ha $\left.^{-1}\right)$, Dash $\mathrm{HC}^{\circledR}\left(0,5 \%\right.$ v.v.) e Emultec R $\mathrm{R}^{\circledR}$ $\left(0,05 \mathrm{~L} \mathrm{ha}^{-1}\right)$. Os adjuvantes foram adicionados à calda de aplicação juntamente com o herbicida Roundup Transorb $\mathrm{R}^{\circledR}$ na dose de 2,16 kg e.a. ha- ${ }^{-1}$. Além desses, foram incluídos dois tratamentos testemunha: um sem aplicação e outro com aplicação de glyphosate sem adjuvante. As variáveis avaliadas foram a distância percorrida pelas gotas para fora do alvo, a fitotoxicidade causada pela deriva, o diâmetro mediano volumétrico, a densidade de gotas e o volume recuperado pela utilização de papéis hidrossensiveis. As pontas de pulverização TTI 110.015 e AIXR 110.015 proporcionaram menor deriva que a ponta XR 110.015. As pontas TTI e AIXR apresentaram menor densidade de gotas e maior diâmetro mediano volumétrico, quando comparadas à ponta XR. A utilização dos adjuvantes NP- $10^{\circledR}$ e Emultec $\mathrm{R}^{\circledR}$ com a ponta de pulverização XR 110.015 aumentou a deriva quando comparada com a aplicação de glyphosate sem adjuvante, porém não diferiram dos demais adjuvantes. O adjuvante Emultec $\mathrm{R}^{\circledR}$ aumentou o DMV das gotas, quando geradas pela ponta XR 110.015. A utilização dos adjuvantes Grap' Oil ${ }^{\circledR}$, Grap Super Gun ${ }^{\circledR}$, Iharol ${ }^{\circledR}$ e Dash $\mathrm{HC}^{\circledR}$ diminuiu a densidade de gotas derivadas, quando geradas pela ponta XR 110.015.
\end{abstract}

Palavras-chave: tecnologia de aplicação, espectro de gotas, pulverização terrestre, fitotoxicidade.

ABSTRACT - Glyphosate drift can cause environmental impact and also affect crops that are sensitive to this herbicide. The aim of this study was to evaluate the influence of different adjuvants and different nozzles on glyphosate drift in terrestrial applications. The study was conducted in the field, arranged in a factorial design (3 $x$ 9): factor A consisted of three different spray nozzles: one flat spray nozzle (Teejet $=X R$ 110.015) and two air induction flat spray nozzles (Teejet AIXR 110.015 and Teejet TTI 110.015). Factor B was composed of seven adjuvants: Break Thru (O.1\% V.v.),

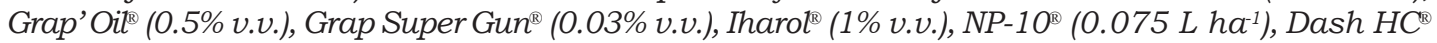
(0.5\% v.v.) and Emultec $R^{\circledR}\left(0.05 \mathrm{~L} \mathrm{ha}^{-1}\right)$. The adjuvants were added to Roundup Transorb $R^{\circledR}$ herbicide at 2.16 a.i. $h a^{-1}$. Moreover, there were two control treatments, one untreated check and another one with application of glyphosate without adjuvant. The distance of droplets off-target, injury caused by drift, volumetric median diameter (VMD), droplet density and volume were evaluated by water sensitive paper cards. The TTI 110.015 spray nozzle resulted in lesser drift when compared to the AIXR 110.015 and XR 110.015 nozzles. The TTI and AIXR nozzles showed lower droplet density and greater VMD compared with the XR nozzle. The use of $N P-1 O^{\circledR}$ and Emultec $R^{\circledR}$ adjuvants with spray nozzle XR 110.015 increases drift. Adjuvant Emultec $R^{\circledR}$ increases VMD droplets when used with the XR 110.015 nozzle. The use of adjuvants Grap' Oil ${ }^{\mathbb{B}}$, Grap Super Gun ${ }^{\mathbb{B}}$, Iharol ${ }^{\mathbb{B}}$ and Dash $H C^{\circledR}$ decrease density derived droplets when generated with the XR 110.015 nozzle.

Keywords: application technology, droplet spectrum, terrestrial spraying, phytotoxicity.

Recebido para publicação em 14.7.2014 e aprovado em 20.11.2014.

2 Universidade Federal de Pelotas - UFPel, Pelotas-RS, Brasil; <laavilabr@gmail.com>. 


\section{INTRODUÇÃO}

Pulverizadores autopropelidos, tratorizados e aviões agrícolas são os principais equipamentos utilizados para aplicação de herbicidas na agricultura brasileira. Os equipamentos de pulverização têm a função de distribuir o produto com tamanho de gotas adequado sobre o alvo. Para aferir a eficiência da pulverização, é necessário determinar características como diâmetro mediano, uniformidade do tamanho e densidade das gotas e, ainda, a cobertura da pulverização (Ozmeri \& Cilingir, 1992).

A rigor, gotas menores melhoram a deposição do produto no alvo, proporcionando melhores resultados de controle (Schröder, 2003). Entretanto, a utilização de gotas menores pode implicar perdas por evaporação e deriva. Já gotas maiores tendem a se depositar na parte superior das plantas (Kirk et al., 1992). Contudo, a uniformidade de distribuição está intimamente relacionada à regulagem adequada do equipamento, à velocidade de trabalho e à escolha das pontas. Dessa forma, estudos relacionados com a tecnologia de aplicação tornam-se importantes para aumentar a eficiência e distribuição de gotas no alvo.

As pontas de pulverização representam um dos principais componentes da aplicação, garantindo a qualidade e a segurança desta. Elas apresentam como funções básicas: fragmentar o liquido em pequenas gotas, distribuir as gotas e controlar a saída do líquido por unidade de área (Sidahmed, 1998). Assim, a seleção das pontas é de suma importância, pois é o fator determinante da quantidade aplicada por área, da uniformidade de aplicação, da cobertura obtida e do risco potencial de deriva.

$\mathrm{Na}$ atualidade, diversas pontas de pulverização estão sendo comercializadas, porém as mais usadas são as de jato plano, que podem apresentar risco de deriva conforme o ângulo de abertura de leque e a pressão de trabalho (Cunha et al., 2003). No entanto, com o desenvolvimento de pontas antideriva, há tendência de substituição das que eram rotineiramente utilizadas por aquelas com indução de ar, pois o potencial risco de deriva é menor. Essas pontas de pulverização são dotadas de préorificio (indutor de ar), localizado antes da abertura para a formação do jato, que permite a formação de gotas de maior diâmetro (Cunha et al., 2003).

A deriva está principalmente relacionada ao tamanho de gotas e à velocidade do vento. Ela é definida como a quantidade de agrotóxicos desviada para fora do alvo a ser atingido, por correntes de ar durante ou depois das aplicações (Miller, 1993). Esse transporte pode ser na forma de gotas ou vapor, sendo a segunda dependente da pressão de vapor e das características físico-químicas da formulação (Miller, 2004). Gotas finas, cujo diluente é água, possuem tempo de vida pequeno e, se esse diluente do produto químico evaporar, o tamanho da gota diminui, possibilitando o carregamento dessa partícula por uma distância muito grande pelas correntes de ar (Matthews, 2000). Assim, em temperaturas elevadas e baixa umidade relativa do ar, deve ser considerada a adição de adjuvantes à calda, para aumentar o tempo de vida da gota, diminuindo a deriva.

O acréscimo de adjuvante pode alterar o padrão de gotas. As mudanças nas propriedades do líquido pulverizado podem influenciar tanto o processo de formação das gotas como o comportamento destas em contato com o alvo, alterando o risco potencial de deriva da aplicação (Miller \& Ellis, 2000). Os adjuvantes são produtos adicionados ao tanque de pulverização com o objetivo de modificar a ação química e as propriedades físicas da calda (Hazen, 2000). A formação da gota é resultado da interação entre a ponta e o líquido de pulverização; assim, o desempenho da ponta é suscetivel de ser afetado pelas propriedades dos líquidos e pela adição de adjuvantes (De Ruiter, 2002).

Um simples adjuvante pode não desempenhar todas essas funções, mas diferentes adjuvantes compativeis frequentemente são combinados para desempenhar múltiplas funções simultaneamente (Stock \& Briggs, 2000). Os adjuvantes podem ser designados para desempenhar funções específicas, incluindo tamponantes, dispersantes, emulsificantes, molhantes, adesivos e espalhantes. Os adjuvantes também podem reduzir a evaporação, espuma, volatilização e deriva.

O êxito das pulverizações é dependente ainda das condições ambientais. Temperatura 
e umidade relativa do ar alteram a evaporação das gotas, recomendando-se evitar operações com temperaturas superiores a $30^{\circ} \mathrm{C}$ e umidade inferior a 55\% (Sosbai, 2012). A velocidade do vento influi diretamente sobre a deriva de gotas, que é o arraste lateral. O ideal é que as aplicações sejam realizadas com ventos até $10 \mathrm{~km} \mathrm{~h}^{-1}$ e direção constante, pois a ausência de vento favorece a formação de correntes ascendentes e velocidades superiores a $10 \mathrm{~km}$ $\mathrm{h}^{-1}$ oferecem maior risco de deriva. No entanto, em regiões litorâneas é frequente a necessidade de operar com ventos mais intensos, limitando-se a recomendação na velocidade de $15 \mathrm{~km} \mathrm{~h}^{-1}$. Esse monitoramento das condições ambientais deve ser realizado pelos técnicos executores ou operadores durante as aplicações com termohigroanemômetros (Sosbai, 2012).

Em vista do exposto, faz-se necessário estudar a redução da deriva pela aplicação de agrotóxicos em ambientes agrícolas. Dessa forma, o objetivo do estudo foi avaliar a influência de adjuvantes e pontas de pulverização na deriva de glyphosate aplicado via terrestre.

\section{MATERIAL E MÉTODOS}

O estudo foi conduzido em campo durante o ano agrícola 2013/14. Os tratamentos foram arranjados em esquema fatorial ( $3 \times 9)$, sendo o primeiro fator constituído por três diferentes pontas de pulverização: uma de jato plano (Teejet XR 110.015) e duas de jato plano com indução de ar (Teejet AIXR 110.015 e Teejet TTI 110.015). O segundo fator foi composto por sete adjuvantes: Break Thru ${ }^{\circledR}(0,1 \%$ v.v.), Grap' $\mathrm{Oil}^{\circledR}\left(0,5 \%\right.$ v.v.), Grap Super Gun ${ }^{\circledR}(0,03 \%$ v.v.), Iharol ${ }^{\circledR}(1 \%$ v.v. $)$, NP- $10^{\circledR}\left(0,075 \mathrm{~L} \mathrm{ha}^{-1}\right)$, Dash $\mathrm{HC}^{\circledR}\left(0,5 \%\right.$ v.v.) e Emultec $\mathrm{R}^{\circledR}\left(0,05 \mathrm{~L}^{\mathrm{h}} \mathrm{a}^{-1}\right)$, descritos na Tabela 1 . Os adjuvantes foram adicionados à calda de aplicação juntamente com o herbicida Roundup Transorb $\mathrm{R}^{\circledR}$ na dose de 2,16 kg e.a. ha-1. Além desses, havia dois tratamentos testemunha: um sem aplicação e outro com aplicação de somente glyphosate. O delineamento experimental realizado foi em blocos casualizados com três repetições.

As unidades experimentais foram compostas por parcelas de diferentes dimensões, definidas por experimento prévio realizado na safra 2012/13. Para a ponta de pulverização Teejet XR 110.015, as unidades experimentais foram compostas por parcelas com $3 \times 11 \mathrm{~m}$; para a ponta Teejet AIXR 110.015, por parcelas com $3 \times 7 \mathrm{~m}$; e para a ponta Teejet TTI 110.015, por parcelas com 3 x $6 \mathrm{~m}$. Foram utilizadas parcelas de dimensões diferentes devido às características referentes às pontas de pulverização, visto que para pontas com indução de ar as perdas por deriva são menores, o que possibilita o menor comprimento da parcela. O cultivar de arroz irrigado semeado na área foi o Puitá INTA-CL, na

Tabela 1 - Descrição dos adjuvantes utilizados no experimento, conforme informações das bulas e rótulos dos produtos

\begin{tabular}{|c|c|c|c|}
\hline Nome comercial & Composição & Função & Formulação \\
\hline Break Thru & $\begin{array}{l}\text { Copolímero poliéter - polimetil } \\
\text { siloxano (silicone) } 100 \%\end{array}$ & Espalhante não iônico & Concentrado solúvel \\
\hline Grap' Oil & Óleo vegetal & $\begin{array}{c}\text { Adjuvante, espalhante adesivo } \\
\text { e antievaporante }\end{array}$ & Óleo emulsionável \\
\hline Grap Super Gun & $\begin{array}{c}\text { Ácido fosfórico, ureia, álcool laurílico } \\
\text { etoxilado }\end{array}$ & Adjuvante & $\mathrm{ND}^{1 /}$ \\
\hline Iharol & $\begin{array}{c}\text { Hidrocarbonetos parafínicos, ciclo } \\
\text { parafínico e aromáticos saturados e } \\
\text { insaturados provenientes da destilação } \\
\text { do petróleo (Óleo mineral) }\end{array}$ & $\begin{array}{c}\text { Adjuvante, inseticida e } \\
\text { acaricida }\end{array}$ & Concentrado emulsionável \\
\hline NP-10 & Nitrogênio e fósforo & Fertilizante foliar & $\mathrm{ND}^{1 /}$ \\
\hline Dash HC & $\begin{array}{l}\text { Hidrocarboneto aromático, ésteres } \\
\text { metílicos, ácido graxo insaturado e } \\
\text { tensoativo }\end{array}$ & Adjuvante & Concentrado emulsionável \\
\hline Emultec R & Nitrogênio e fósforo & Fertilizante foliar & $\mathrm{ND}^{1 /}$ \\
\hline
\end{tabular}

\footnotetext{
1/ Não disponibilizado pelo fabricante.
} 
densidade de $100 \mathrm{~kg}$ de sementes ha ${ }^{-1}$; ele foi usado como planta bioindicadora para mensurar a deriva. As sementes foram previamente tratadas com o inseticida fipronil e fungicida carboxi-thiran nas doses de $37,5 \mathrm{e}$ 50 g i.a. por $100 \mathrm{~kg}$ de sementes, respectivamente. A adubação de base foi feita com $300 \mathrm{~kg} \mathrm{ha}^{-1}$ da fórmula 05-20-20. A adubação nitrogenada de cobertura foi realizada com ureia (46-00-00), utilizando $46 \mathrm{~kg} \mathrm{ha}^{-1}$ de $\mathrm{N}$, no estádio $\mathrm{V}_{4}$, após a aplicação dos tratamentos e antes da entrada de água.

Os tratamentos foram aplicados com pulverizador costal de precisão $\left(\mathrm{CO}_{2}\right)$. A barra de aplicação utilizada tinha $2 \mathrm{~m}$, com quatro pontas espaçadas de $50 \mathrm{~cm}$, calibradas para aplicar um volume de calda de $150 \mathrm{~L} \mathrm{ha}^{-1}$. A fim de realizar a aplicação com iguais condições climáticas para cada tratamento, utilizaram-se três pulverizadores, os quais foram aferidos e calibrados previamente com pressão de trabalho de $171 \mathrm{kPa}$. Para minimizar erros durante a aplicação, os aplicadores acertaram a distância do passo e a velocidade de deslocamento em $1 \mathrm{~m} \mathrm{~s}^{-1}$. Durante a aplicação também foi utilizado metrômetro digital Seiko ${ }^{\circledR}$ modelo DM 50, o qual foi regulado para a cada segundo realizar um aviso sonoro. A aplicação dos tratamentos foi feita quando a cultura se encontrava no estádio $\mathrm{V}_{4}$, segundo a escala fenológica descrita por Counce et al. (2000).

As avaliações de deriva foram feitas observando-se a distância percorrida pelas gotas para fora do local de aplicação, através da fitotoxicidade causada pelo herbicida e pela utilização de papéis hidrossensiveis posicionados verticalmente. A distância percorrida foi calculada visualmente através da fitotoxicidade aos 21 dias após a aplicação do herbicida, com uso de trena na direção em que o vento se encontrava no momento da aplicação. A parcela foi dividida a cada metro, na direção da deriva, com a finalidade de criar gradiente de fitotoxicidade, em que se atribuíram notas percentuais. A escala variou de zero a $100 \%$, em que zero corresponde à ausência de sintomas de fitotoxicidade e $100 \%$ à morte das plantas de arroz (Gazziero et al., 1995).

Utilizaram-se papéis hidrossensiveis para avaliar o diâmetro mediano volumétrico, a densidade de gotas e o volume recuperado.
Foram usados dois papéis hidrossensiveis por parcela: um foi colocado horizontalmente em superfície fixa no topo da cultura, e o outro, colocado verticalmente $50 \mathrm{~cm}$ acima do topo da cultura, distante $50 \mathrm{~cm}$ da extremidade da faixa de aplicação.

Após a aplicação de cada tratamento, os papéis foram retirados, identificados e armazenados em ambiente hermético, sendo posteriormente enviados para laboratório, onde foram analisados com o software Agroscan ${ }^{\circledR}$. Esse software analisa as gotas das pulverizações agrícolas utilizando digitalização de imagens dos cartões hidrossensiveis, o que possibilitou determinar as características das gotas aspergidas e derivadas, como diâmetro mediano volumétrico, densidade de gotas e volume recuperado.

As condições climáticas foram monitoradas durante todo o período da aplicação, com o uso de dois termohigroanemômetros das marcas Kestrel $4000^{\circledR}$ e Icel $^{\circledR}$, os quais foram aferidos antes da utilização. Um dos equipamentos foi instalado próximo à área onde se realizou o experimento, o qual armazenou os dados de umidade relativa do ar, temperatura do ambiente, velocidade e direção do vento, a cada 20 segundos. Já o outro foi utilizado manualmente durante a pulverização, monitorando as possiveis variações que ocorressem durante a execução do trabalho, garantindo que a aplicação dos tratamentos fosse realizada com parâmetros meteorológicos semelhantes.

Após as avaliações, os dados coletados foram analisados quanto à sua normalidade e homocedasticidade, quando necessário, os dados foram transformados por $Y t=\operatorname{arcseno} \sqrt{(y=0,5) / 100}$. Logo, todas as variáveis foram submetidas à análise de variância $(\mathrm{p} \leq 0,05)$. Para a variável fitotoxicidade, realizou-se análise trifatorial, em que o fator $\mathrm{C}$ constituiu-se das distâncias de avaliação (1, $2,3,4,5,6,7,8$ e 9 metros). Em caso de significância, as médias dos tratamentos foram comparadas pelo teste de Tukey $(\mathrm{p} \leq 0,05)$.Também se realizou a análise de correlação de Pearson entre as variáveis distância percorrida pela deriva e densidade de gotas, bem como com diâmetro mediano volumétrico. 


\section{RESULTADOS E DISCUSSÃO}

No momento da aplicação de cada tratamento, as velocidades de vento registradas foram inferiores a $14 \mathrm{~km} \mathrm{~h}^{-1} \mathrm{e}$ a direção do vento manteve-se constante (Figura 1). As condições climáticas ideais para aplicação de agrotóxicos são umidade relativa superior a $50 \%$, temperatura do ar até $30{ }^{\circ} \mathrm{C}$ e velocidade do vento até $10 \mathrm{~km} \mathrm{~h}^{-1}$ (Antuniassi, 2012). Contudo, tratando-se de uma região litorânea, há necessidade, muitas vezes, de operar com ventos mais intensos, limitando-se a recomendação na velocidade de $15 \mathrm{~km} \mathrm{~h}^{-1}$ (SOSBAI, 2012).

Os dados obtidos atenderam às pressuposições da análise de variância, com interação entre os tratamentos pontas de pulverização e adjuvantes para as variáveis analisadas. Entre as pontas avaliadas, de modo geral, a XR 110.015 foi a que proporcionou maior deriva, seguida da AIXR 110.015 e TTI 110.015, independentemente do adjuvante utilizado (Tabela 2), o que está de acordo com os resultados obtidos por Moreira Júnior (2009), que observou os maiores niveis de deriva com a utilização da ponta XR 80.03. Provavelmente isso acontece devido ao fato de a ponta XR 110.015 não possuir indução de ar, resultando em gotas de menor diâmetro (Tabela 3), o que possibilita maior densidade de gotas (Tabela 4), e em melhor cobertura do alvo, embora estejam sujeitas ao maior arraste pelo vento para fora da área de aplicação.

Ao comparar os adjuvantes aplicados com a ponta XR 110.015, foi possivel observar que não houve redução na distância de deriva ao utilizá-los na calda de aplicação. Ainda considerando a ponta XR 110.015, a maior deriva foi observada com o uso dos adjuvantes Emultec $\mathrm{R}^{\circledR}$ e $\mathrm{NP}-10^{\circledR}$, diferindo estatisticamente da aplicação do tratamento somente com glyphosate, porém não dos tratamentos com os demais adjuvantes (Tabela 2). Esses resultados corroboram o obtido por Santos (2007), o qual, ao comparar niveis de deposição de adjuvantes por aplicação via aérea, concluiu que, ao usar o adjuvante Emultec plus ${ }^{\circledR}$, houve menor deposição nos coletores, caracterizando maior perda por deriva. Resultados semelhantes

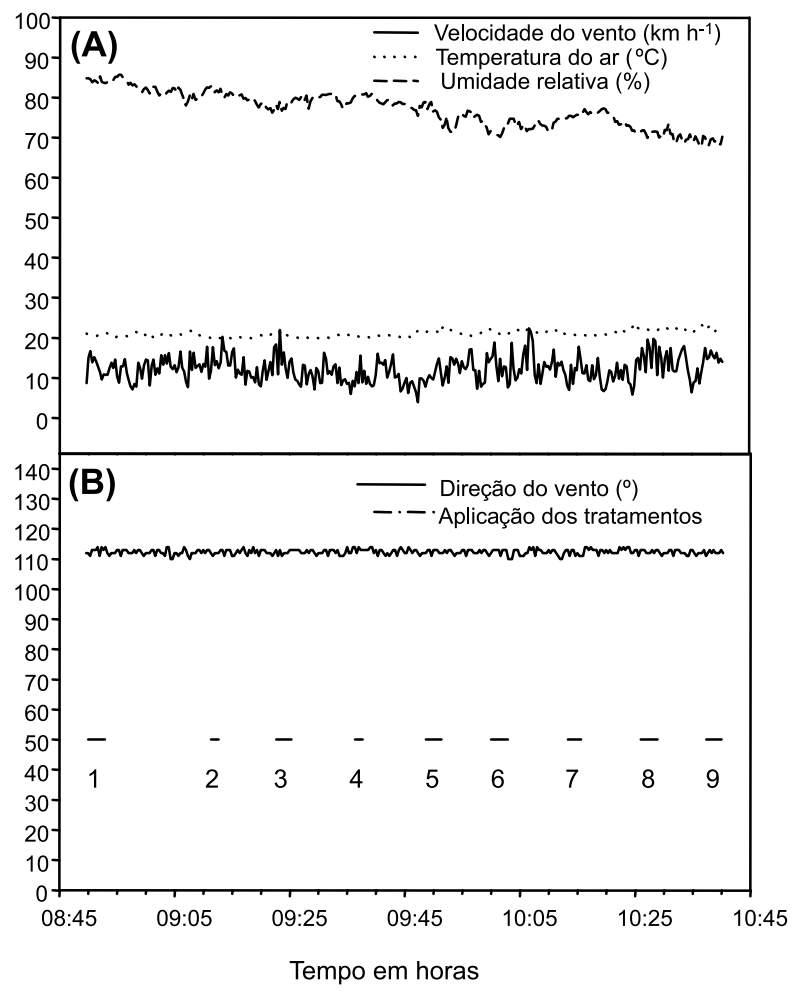

Figura 1 - Velocidade do vento, umidade relativa do ar, temperatura do ar (A) e direção do vento (B) durante o período de aplicação no dia 22 de novembro de 2013. Testemunha com aplicação de água (1), Glyphosate (2), Gly+Break Thru (3), Gly+Grap'Oil (4), Gly+Grap Super Gun (5), Gly+Iharol (6), Gly+NP-10 (7), Gly+Dash (8) e Gly+Emultec R (9).

Tabela 2 - Distância (m) da deriva de glyphosate, a partir da lateral da parcela pulverizada, avaliada através da fitotoxicidade em plantas de arroz irrigado aos 21 dias após a aplicação dos tratamentos, em função da ponta de pulverização e de adjuvantes

\begin{tabular}{|c|c|c|c|}
\hline \multirow{2}{*}{ Adjuvantes } & \multicolumn{3}{|c|}{ Deriva $(\mathrm{m})$} \\
\hline & XR 110.015 & AIXR 110.015 & TTI 110.015 \\
\hline Gly. ${ }^{1 /}$ & $4,33 \mathrm{~b} \mathrm{~A}^{-3 /}$ & $2,93^{\mathrm{ns} 4 /} A B$ & $1,27^{\mathrm{ns}} \mathrm{B}$ \\
\hline Break Thru ${ }^{2 /}$ & $5,57 \mathrm{ab} A$ & $4,20 \mathrm{AB}$ & $1,87 \mathrm{~B}$ \\
\hline Grap'Oil ${ }^{2 /}$ & $6,83 \mathrm{ab} \mathrm{A}$ & $2,26 \mathrm{~B}$ & $1,73 \mathrm{~B}$ \\
\hline Grap Super Gun & $7,00 \mathrm{ab} A$ & $2,90 \mathrm{~B}$ & $1,40 \mathrm{~B}$ \\
\hline Iharol $^{2 /}$ & $5,50 \mathrm{ab} A$ & $3,93 \mathrm{~B}$ & $2,07 \mathrm{~B}$ \\
\hline $\mathrm{NP}-10^{2 /}$ & 8,16 a A & $3,00 \mathrm{~B}$ & $2,17 \mathrm{~B}$ \\
\hline Dash $\mathrm{HC}^{\underline{2} /}$ & $6,00 \mathrm{ab} \mathrm{A}$ & $2,73 \mathrm{~B}$ & $1,57 \mathrm{~B}$ \\
\hline Emultec $\mathrm{R}^{2 /}$ & 9,00 a A & $3,03 \mathrm{~B}$ & $3,00 \mathrm{~B}$ \\
\hline
\end{tabular}

1/ Testemunha aplicada somente com glyphosate. ${ }^{2 /}$ Todos os adjuvantes foram aplicados juntamente com glyphosate. ${ }^{3} /$ Médias seguidas por letras minúsculas iguais na coluna e letras maiúsculas iguais na linha não diferem entre si pelo teste de Tukey a 5\% de probabilidade. ${ }^{4 /}$ Não significativo.

Planta Daninha, Viçosa-MG, v. 33, n. 2, p. 375-386, 2015 
foram obtidos ao avaliar perdas por deriva dos adjuvantes In-Tec ${ }^{\circledR}$, Li $700^{\circledR}$ e óleo vegetal aplicados com ponta de jato plano; esse autor observou que o uso de óleo vegetal (0,6\% v.v) e dos adjuvantes In-Tec ${ }^{\circledR}$ e Li $700^{\circledR}$ resultou nas maiores perdas. Desses adjuvantes, o Li $700^{\circledR}$ também é comercializado como redutor de deriva (Chechetto \& Antuniassi, 2012).

Tabela 3 - Diâmetro mediano volumétrico $(\mu \mathrm{m})$ observado no alvo de aplicação pelos tratamentos aplicados por pulverizador terrestre e coletados com cartão hidrossensível

\begin{tabular}{|c|c|c|c|}
\hline \multirow{2}{*}{ Adjuvantes } & \multicolumn{3}{|c|}{$\mathrm{DMV}(\mu \mathrm{m})$} \\
\hline & XR 110.015 & AIXR 110.015 & TTI 110.015 \\
\hline Test. ${ }^{1 /}$ & $643 \mathrm{~b} \mathrm{~B}^{4 /}$ & $1170^{\mathrm{ns} 5 /} \mathrm{AB}$ & $23800^{\text {ns }} \mathrm{A}$ \\
\hline Gly. $\stackrel{2 /}{ }$ & $516 \mathrm{~b} \mathrm{~B}$ & $1663 \mathrm{~A}$ & $1814 \mathrm{~A}$ \\
\hline Break Thru ${ }^{3 /}$ & $818 \mathrm{~b} \mathrm{~B}$ & $1977 \mathrm{~A}$ & $2013 \mathrm{~A}$ \\
\hline Grap'Oil $^{3 /}$ & $957 \mathrm{~b} \mathrm{~B}$ & $2070 \mathrm{~A}$ & $2380 \mathrm{~A}$ \\
\hline Grap Super Gun ${ }^{3 /}$ & $712 b^{n s}$ & 1068 & 1359 \\
\hline Iharol $^{3 /}$ & $673 \mathrm{~b} \mathrm{~B}$ & $1533 \mathrm{~A}$ & $1713 \mathrm{~A}$ \\
\hline $\mathrm{NP}-10^{\frac{3}{}}$ & $713 \mathrm{~b} \mathrm{~B}$ & $1476 \mathrm{AB}$ & $2247 \mathrm{~A}$ \\
\hline Dash $\mathrm{HC}^{\underline{3} /}$ & $857 \mathrm{~b} \mathrm{~B}$ & $1670 \mathrm{AB}$ & $2395 \mathrm{~A}$ \\
\hline Emultec $\mathrm{R}^{\frac{3}{} /}$ & $1022 a^{n s}$ & 1316 & 1983 \\
\hline
\end{tabular}

${ }^{1 /}$ Testemunha com aplicação de água. 르 Testemunha aplicada somente com glyphosate. ${ }^{3 /}$ Todos os adjuvantes foram aplicados juntamente com glyphosate. 4/ Médias seguidas por letras minúsculas iguais na coluna e letras maiúsculas iguais na linha não diferem entre si pelo teste de Tukey a $5 \%$ de probabilidade. ${ }^{5 /}$ Não significativo.

Tabela 4 - Densidade de gotas $\left(\right.$ gotas $\mathrm{cm}^{-2}$ ) geradas pelos tratamentos aplicados por via terrestre e coletadas com cartão hidrossensível posicionado horizontalmente

\begin{tabular}{|c|c|c|c|}
\hline \multirow{2}{*}{ Adjuvantes } & \multicolumn{3}{|c|}{ Densidade $\left(\right.$ gotas $\mathrm{cm}^{-2}$ ) } \\
\hline & XR 110.015 & AIXR 110.015 & TTI 110.015 \\
\hline Test. $^{1 /}$ & $129 \mathrm{a} \mathrm{A}^{4 /}$ & $38^{\mathrm{ns} 5 /} \mathrm{B}$ & $14^{\mathrm{ns}} \mathrm{B}$ \\
\hline Gly.2/ & $105 \mathrm{ab} \mathrm{A}$ & $68 \mathrm{AB}$ & $29 \mathrm{~B}$ \\
\hline Break Thru ${ }^{3 /}$ & $66 \mathrm{~b} \mathrm{~A}$ & $40 \mathrm{AB}$ & $17 \mathrm{~B}$ \\
\hline Grap'Oil $^{\prime} /$ & $100 \mathrm{ab} \mathrm{A}$ & $42 \mathrm{~B}$ & $35 \mathrm{~B}$ \\
\hline Grap Super Gun ${ }^{3 /}$ & $94 \mathrm{ab} \mathrm{A}$ & $43 \mathrm{~B}$ & $17 \mathrm{~B}$ \\
\hline Iharol $^{3 /}$ & $78 \mathrm{~b} \mathrm{~A}$ & $57 \mathrm{AB}$ & $17 \mathrm{~B}$ \\
\hline $\mathrm{NP}-10^{3 / /}$ & $112 \mathrm{ab} \mathrm{A}$ & $37 \mathrm{~B}$ & $18 \mathrm{~B}$ \\
\hline Dash $\mathrm{HC}^{3 / /}$ & $103 \mathrm{ab} A$ & $33 \mathrm{~B}$ & $14 \mathrm{~B}$ \\
\hline Emultec $\mathrm{R}^{3 /}$ & $92 \mathrm{ab} \mathrm{A}$ & $37 \mathrm{~B}$ & $15 \mathrm{~B}$ \\
\hline
\end{tabular}

${ }^{1 /}$ Testemunha com aplicação de água. 르 Testemunha aplicada somente com glyphosate. ${ }^{3 /}$ Todos os adjuvantes foram aplicados juntamente com glyphosate. ${ }^{4 /}$ Médias seguidas por letras minúsculas iguais na coluna e letras maiúsculas iguais na linha não diferem entre si pelo teste de Tukey a $5 \%$ de probabilidade. ${ }^{5 /}$ Não significativo.
Em se tratando dos tratamentos aplicados com as pontas AIXR 110.015 e TTI 110.015, observou-se que não houve diferença significativa na redução da deriva da aplicação de glyphosate ao se utilizarem os diferentes adjuvantes, demonstrando que, para esses tipos de pontas de pulverização, os adjuvantes tiveram comportamento semelhante ao do tratamento com glyphosate sem adjuvante (Tabela 2). Dessa forma, pode-se concluir que, para muitos adjuvantes, existe defasagem entre o que está descrito na especificação técnica ou no posicionamento dos fabricantes e as reais funções observadas (Oliveira \& Antuniassi, 2012).

Esses resultados corroboram os de Cunha et al. (2003), os quais concluíram que pontas de jato plano sem indução de ar podem apresentar maior risco de deriva, dependendo do ângulo de abertura do leque e da pressão de trabalho utilizada. Na mesma linha, em trabalho realizado em túnel de vento com objetivo de avaliar pontas de pulverização, verificou-se menor ocorrência de deriva ao utilizar pontas com indução de ar (AI), quando comparadas com as pontas de modelos convencionais (XR) (Fietsam et al., 2004). Foram observadas menores perdas por deriva para pontas com indução de ar, em comparação com pontas de jato plano convencional, jato cônico vazio e jato plano com pré-orifício, sob diferentes condições ambientais e pressão de trabalho (Costa et al., 2007). Esses resultados estão de acordo também com os obtidos em campo, onde se observaram valores reduzidos de deriva para pontas com indução de ar (Nuyttens et al., 2006).

As demais pontas de pulverização testadas são denominadas de antideriva, pois possuem pré-orificio (indutor de ar), localizado antes da abertura para a formação do jato, o que permite gerar gotas de maior diâmetro, reduzindo assim o potencial de risco de deriva, como observado no presente trabalho. Segundo Miller (2004), pontas com indução de ar reduzem a perda por deriva em $75 \%$, em relação a sistemas convencionais para pulverizadores de barra. O pré-orificio produz perda de carga na pressurização, causando efeito inverso no diâmetro das gotas (Cunha et al., 2007). Contudo, comparando-se diferentes pontas antideriva, estas podem gerar espectros 
de gotas distintos, o que pode explicar as diferenças que ocorreram no presente trabalho, estando de acordo com o que foi descrito por Piggott \& Matthews (1999), os quais afirmam que nem todas as pontas de pulverização com indução de ar respondem da mesma forma ao considerar o espectro de gotas gerado.

$\mathrm{O}$ diâmetro mediano volumétrico (DMV) das pontas AIXR e TTI não diferiu entre si (Tabela 3), porém, de modo geral, o espectro de gotas gerado por essas pontas é diferente, o que corrobora os resultados obtidos por Miller $\&$ Butler Ellis (2000), que, ao estudarem características da pulverização resultante do efeito das formulações, concluíram que pontas com indução de ar são mais sensíveis às mudanças das características físicas das caldas. O comportamento das pulverizações com esses tipos de ponta não segue um mesmo padrão em relação às pontas hidráulicas convencionais, fato esse confirmado por Cunha et al. (2003), ao avaliarem estratégias de redução de deriva de agrotóxicos.

As pontas de pulverização com indução de ar produziram gotas com maior diâmetro mediano volumétrico (DMV) quando comparadas com a ponta sem indução de ar (XR). Esse resultado está de acordo com os obtidos por Derksen et al. (1999), os quais afirmam que pontas com indução de ar produzem maior diâmetro mediano volumétrico, o que resulta em menor transporte das gotas pulverizadas para fora da área alvo. De modo geral, a ponta XR 110.015 gerou menor DMV quando comparada com as pontas AIXR 110.015 e TTI 110.015, para todos adjuvantes utilizados, diferindo significativamente entre si, com exceção da testemunha e dos tratamentos em que foram utilizados NP-10 ${ }^{\circledR}$ e Dash $^{\circledR}$ (Tabela 3). Esses resultados corroboram os obtidos por Chechetto \& Antuniassi (2012), que, ao avaliarem o DMV de duas pontas de pulverização, uma de jato plano com pré-orificio e outra de jato plano com indução de ar, concluíram que o maior diâmetro das gotas é naquela que possui o orificio indutor de ar.

No entanto, os valores muito altos do DMV não eram esperados, pois não estão de acordo com a característica das pontas utilizadas. Há duas possiveis explicações. A primeira pode estar relacionada ao fato de que o software que analisa as gotas esteja calibrado para água pura, portanto, em caldas aquosas, os valores são mais confiáveis. Quando as gotas geradas são provenientes de calda com adjuvantes, estes podem aumentar o contato das gotas com o papel, espalhando-as em uma área amostral maior, superestimando o DMV, o que corrobora os resultados observados por Costa (2009). Além disso, o sistema pode não identificar as gotas coalescentes, como as gotas individuais, e sim como gota única, com o diâmetro muito maior que o real (Schröder \& Loeck, 2006).

Ao considerar as pontas de pulverização AIXR e TTI, não houve diferença no DMV entre os diferentes tratamentos com adjuvantes (Tabela 3). Para a ponta XR, os adjuvantes não diferiram significativamente da testemunha e do tratamento aplicado somente com glyphosate, com exceção do adjuvante Emultec $R^{\circledR}$, o qual diferiu estatisticamente dos demais e apresentou o maior DMV. Esperavase que, ao adicionar adjuvante à calda de aplicação, fosse alterado o DMV das gotas produzidas pelas pontas AIXR e TTI, o que não ocorreu. Resultados semelhantes foram obtidos por Sanderson et al. (1997), os quais, ao adicionarem óleo vegetal e mineral à calda de aplicação, de modo geral, obtiveram aumento do diâmetro das gotas aspergidas, porém sem diferença dos demais tratamentos realizados pelos autores.

Pontas sem indução de ar geram maior densidade de gotas, como observado; de modo geral, a ponta XR apresentou maior densidade de gotas que as pontas AIXR e TTI (Tabela 4).

Essa diferença na densidade de gotas já era esperada em razão das características técnicas das pontas utilizadas, corroborando com os resultados obtidos por Viana et al. (2008), ao avaliarem a densidade de gotas depositadas no topo da cultura da soja por diferentes pontas de pulverização, verificaram que a ponta XR 110.02 resultou em maior densidade, juntamente com as pontas TXA 80.02 e TJ60 80.02, diferindo estatisticamente da ponta TT 110.02. Isso foi confirmado por Gulart et al. (2013), que, ao aplicarem fungicida e avaliarem a densidade de gotas no terço médio das plantas de trigo, constataram que as maiores densidades depositadas nos coletores foram observadas ao se utilizar a ponta XR 110.02, seguida das pontas TX 80.02 e TJ60 110.02, diferindo estatisticamente entre si. 
Ao se considerar a utilização dos adjuvantes, não houve efeito desses produtos na densidade de gotas para a ponta XR (estatisticamente, houve diferença entre a testemunha e o Break Thru e Iharol para esta ponta), e não houve no uso dos adjuvantes para as pontas com indução de ar.

No entanto, considerando a densidade de gotas coletada no papel hidrossensivel disposto no sentido vertical para avaliar a deriva (Tabela 5), observa-se que, para a ponta XR, o adjuvante Emultec $R^{\circledR}$ foi o que mostrou maior densidade de gotas, juntamente com NP- $10^{\circledR} \mathrm{e}$ Break Thru ${ }^{\circledR}$; isso ajuda a explicar a maior distância percorrida pela deriva nesses tratamentos. A adição de Emultec $\mathrm{R}^{\circledR}$ altera as características da calda de aplicação, resultando em espectro de gotas muito heterogêneo, pois alta densidade de gotas foi detectada no coletor disposto verticalmente, o que resultou no maior volume recuperado para essa ponta, não diferindo somente do adjuvante NP-10 ${ }^{\circledR}$ (Tabela 6), o qual também é comercializado como antideriva.

Ao comparar as pontas de pulverização para avaliação da densidade de gotas depositadas no coletor posicionado verticalmente, utilizando os adjuvantes Grap Super Gun ${ }^{\circledR}$ e NP- $10^{\circledR}$, houve diferença para a ponta XR, que apresentou maior densidade que as demais pontas, e elas não diferiram entre si para essa variável. Ao considerar os adjuvantes utilizados na ponta de pulverização XR, observa-se que Emultec $\mathrm{R}^{\circledR}, \mathrm{NP}-10^{\circledR}$ e Break $\mathrm{Thru}^{\circledR}$ não diferiram estatisticamente da aplicação com glyphosate sem adjuvante, porém diferiram dos demais tratamentos, os quais se comportaram semelhantemente à testemunha, reduzindo a densidade de gotas nesse ponto de coleta.

Resultado semelhante foi obtido por Cunha et al. (2003), que, ao avaliarem a densidade de gotas coletadas em papéis hidrossensiveis, para diferentes pontas de pulverização, com a utilização de óleo vegetal, concluíram que, com o uso do óleo, houve redução significativa na densidade de gotas derivadas do local de aplicação, em comparação à utilização da mesma ponta sem adição de adjuvante. Esse resultado está de acordo com o obtido no presente trabalho, pois, ao se utilizar o óleo vegetal Grap'Oil ${ }^{\circledR}$, houve redução da densidade
Tabela 5 - Densidade de gotas $\left(\right.$ gotas $\mathrm{cm}^{-2}$ ) desviadas $50 \mathrm{~cm} \mathrm{da}$ extremidade da faixa, geradas pelos tratamentos aplicados por pulverização terrestre e coletadas com cartão hidrossensível posicionado verticalmente

\begin{tabular}{|c|c|c|c|}
\hline \multirow{2}{*}{ Adjuvantes } & \multicolumn{3}{|c|}{ Densidade $\left(\right.$ gotas $\left.\mathrm{cm}^{-2}\right)$} \\
\hline & XR 110.015 & AIXR 110.015 & TTI 110.015 \\
\hline Test. $^{\underline{1}}$ & $52 \mathrm{C}^{4 / \mathrm{ns} 5 /}$ & $39^{\mathrm{ns}}$ & $13 \mathrm{ab}$ \\
\hline Gly. ${ }^{2 /}$ & $151 b^{\text {ns }}$ & 78 & $21 \mathrm{ab}$ \\
\hline Break Thru $^{3 /}$ & $167 \mathrm{ab}^{\mathrm{ns}}$ & 123 & $10 \mathrm{ab}$ \\
\hline Grap'Oil $^{3 /}$ & $50 \mathrm{c}^{\mathrm{ns}}$ & 26 & $13 \mathrm{ab}$ \\
\hline Grap Super Gun ${ }^{3 /}$ & $84 \mathrm{c} \mathrm{A}$ & $8 \mathrm{~B}$ & $4 \mathrm{bB}$ \\
\hline Iharol $^{3 /}$ & $48 \mathrm{c}^{\mathrm{ns}}$ & 45 & $21 \mathrm{ab}$ \\
\hline $\mathrm{NP}-10^{3 /}$ & $211 \mathrm{abA}$ & $20 \mathrm{~B}$ & $45 \mathrm{ab} \mathrm{B}$ \\
\hline Dash $\mathrm{HC}^{3 /}$ & $52 c^{\text {ns }}$ & 43 & $41 \mathrm{ab}$ \\
\hline Emultec $\mathrm{R}^{3 /}$ & $341 \mathrm{a}^{\mathrm{ns}}$ & 144 & $71 \mathrm{a}$ \\
\hline
\end{tabular}

1/ Testemunha com aplicação de água. 르 Testemunha aplicada somente com glyphosate. ${ }^{3 /}$ Todos os adjuvantes foram aplicados juntamente com glyphosate. ${ }^{4 /}$ Médias seguidas por letras minúsculas iguais na coluna e letras maiúsculas iguais na linha não diferem entre si pelo teste de Tukey a $5 \%$ de probabilidade. $\underline{5}$ Não significativo.

Tabela 6 - Estimativa do volume recuperado $\left(\mathrm{L} \mathrm{ha}^{-1}\right)$ na área de deriva, coletado em cartão hidrossensível posicionado verticalmente, gerado pelos tratamentos aplicados por via terrestre

\begin{tabular}{|c|c|c|c|}
\hline \multirow{2}{*}{ Adjuvantes } & \multicolumn{3}{|c|}{ Volume recuperado ${ }^{6 /}\left(\mathrm{L} \mathrm{ha}^{-1}\right)$} \\
\hline & XR 110.015 & AIXR 110.015 & TTI 110.015 \\
\hline Test. ${ }^{1 /}$ & $1,4 \mathrm{~b}^{4 / \mathrm{ns} \underline{5} /}$ & $1,1^{\mathrm{ns}}$ & $1,6^{\mathrm{ns}}$ \\
\hline Gly. ${ }^{2 /}$ & $7,2 b^{\text {ns }}$ & 6,2 & 1,1 \\
\hline Break Thru ${ }^{3 /}$ & $10,6 \mathrm{~b} \mathrm{~A}$ & $6,2 \mathrm{~B}$ & $0,4 \mathrm{~B}$ \\
\hline Grap'Oil $^{3 /}$ & $3,0 b^{n s}$ & 2,0 & 0,8 \\
\hline Grap Super Gun ${ }^{3 /}$ & $5,4 \mathrm{~b} \mathrm{~A}$ & $0,2 \mathrm{~B}$ & $0,1 \mathrm{~B}$ \\
\hline Iharol $^{3 /}$ & $2,4 b^{n s}$ & 1,7 & 3,5 \\
\hline $\mathrm{NP}-10^{3 / /}$ & $42,0 \mathrm{a}^{\mathrm{ns}}$ & 6,1 & 3,2 \\
\hline Dash $\mathrm{HC}^{-3 /}$ & $11,9 b^{\text {ns }}$ & 2,4 & 4,7 \\
\hline Emultec $\mathrm{R}^{3 /}$ & $51,2 a^{\text {ns }}$ & 11,1 & 4,8 \\
\hline
\end{tabular}

${ }^{1 /}$ Testemunha com aplicação de água. ${ }^{2 /}$ Testemunha aplicada somente com glyphosate. ${ }^{3 /}$ Todos os adjuvantes foram aplicados juntamente com glyphosate. ${ }^{4 /}$ Médias seguidas por letras minúsculas iguais na coluna e letras maiúsculas iguais na linha não diferem entre si pelo teste de Tukey a $5 \%$ de probabilidade. ${ }^{5 /}$ Não significativo. ${ }^{6 /}$ Calculado pelo software Agroscan ${ }^{\circledR}$.

de gotas derivadas, diferindo significativamente do tratamento com somente glyphosate, bem como dos tratamentos em que se adicionou Emultec $\mathrm{R}^{\circledR}, \mathrm{NP}-10^{\circledR}$ e Break Thru ${ }^{\circledR}$, pulverizados com a ponta Teejet XR 110.015. 
Entretanto, quando se adicionaram adjuvantes na calda de aplicação, utilizando a ponta AIXR, não houve diferença entre os tratamentos. Já para a ponta TTI, não houve efeito dos adjuvantes na densidade de gotas derivadas.

Observou-se que, para o volume de deriva recuperado para a ponta $\mathrm{XR}$, os adjuvantes Emultec $\mathrm{R}^{\circledR}$ e NP-10 ${ }^{\circledR}$, comercializados como antideriva, foram os que mais perderam por arraste das gotas (Tabela 6). Eles diferenciamse estatisticamente dos demais tratamentos, estando de acordo com os dados de distância percorrida pela deriva para fora da área-alvo (Tabela 2), pois foram os adjuvantes que tiveram maior perda da esteira de aplicação, considerando a aplicação com a ponta XR 110.015.

Os valores de volume recuperado estão de acordo com o descrito por Araújo \& Araújo (2012), que, ao analisarem a deposição de gotas por digitalização de imagens, explicaram que a estimativa do volume recuperado de uma aplicação pelo software Agroscan ${ }^{\circledR}$ é feita pela correlação da densidade de gotas com o diâmetro médio delas. Assim, os dados obtidos na análise dos cartões hidrossensiveis estão de acordo com a literatura citada, visto que os adjuvantes Emultec ${ }^{\circledR}$ e NP- $10^{\circledR}$ são os que apresentam maior densidade de gotas recuperadas (Tabela 5) e não diferem estatisticamente dos demais tratamentos quando considerada a avaliação do DMV das gotas derivadas para a ponta XR (Tabela 7).

Considerando o DMV das gotas que derivaram do local de pulverização, pode-se observar que não houve diferença significativa entre as pontas de pulverização, bem como entre os adjuvantes utilizados (Tabela 7), o que possibilita concluir que as pontas de pulverização com indução de ar geram um espectro de gotas muito heterogêneo, porém com menor densidade de gotas que pontas sem o pré-orifício indutor de ar. Isso explica a menor distância percorrida pelas gotas para fora da área-alvo.

O DMV das gotas derivadas do local de aplicação não diferiu entre os tratamentos. Considerando o uso de adjuvantes em cada ponta, observa-se que não houve efeito da sua adição na calda aspergida, com exceção do tratamento em que se utilizou o adjuvante Emultec $\mathrm{R}^{\circledR}$ para a ponta TTI, o qual diferiu da testemunha com aplicação de somente água. Assim, pode-se concluir que as diferentes pontas de pulverização geram um espectro de gota muito heterogêneo, havendo gotas finas e muito finas sendo produzidas, com menor densidade em pontas com indução de ar, e que essas gotas têm comportamento semelhante ao daquelas produzidas em pontas sem indução, quando considerada a sua dispersão no ambiente.

Segundo Cunha et al. (2003), gotas com diâmetro menor que $100 \mu \mathrm{m}$ apresentam alto potencial de deriva, o que está de acordo com o presente estudo. Em trabalho semelhante, Chechetto \& Antuniassi (2012) concluíram que a ponta de jato plano com pré-orificio apresenta espectro de gotas mais sujeito a deriva, em relação à ponta de jato plano com indução de ar, ao realizar avaliação de DMV e pelos valores obtidos de \%vol. $<100 \mu \mathrm{m}$.

De acordo com Chechetto \& Antuniassi (2012), pontas com indução de ar geram espectro de gotas mais heterogêneo quando comparadas com pontas sem indução, o que, de modo geral, está de acordo com os resultados obtidos neste trabalho. Para esses

Tabela 7 - Diâmetro mediano volumétrico $(\mu \mathrm{m})$ das gotas desviadas do alvo por deriva, geradas pelos tratamentos aplicados por pulverização terrestre e coletadas com cartão hidrossensível posicionado verticalmente

\begin{tabular}{|c|c|c|c|}
\hline \multirow{2}{*}{ Adjuvantes } & \multicolumn{3}{|c|}{$\mathrm{DMV}(\mu \mathrm{m})$} \\
\hline & XR 110.015 & AIXR 110.015 & TTI 110.015 \\
\hline Test. ${ }^{1 /}$ & $76 \mathrm{a}^{4 / \mathrm{ns} 5 /}$ & $70 a$ & $63 \mathrm{~b}$ \\
\hline Gly. ${ }^{-/}$ & 89 a A & $120 \mathrm{aA}$ & $93 \mathrm{ab} \mathrm{A}$ \\
\hline Break Thru $^{3 /}$ & $116 \mathrm{a}^{\mathrm{ns}}$ & $100 \mathrm{a}$ & $89 \mathrm{ab}$ \\
\hline Grap'Oil $^{3 /}$ & $91 a^{\text {ns }}$ & $92 \mathrm{a}$ & $121 \mathrm{ab}$ \\
\hline Grap Super Gun ${ }^{3 /}$ & $123 \mathrm{a}^{\mathrm{ns}}$ & $66 \mathrm{a}$ & $87 \mathrm{ab}$ \\
\hline Iharol $^{3 /}$ & $97 a^{\text {ns }}$ & $85 \mathrm{a}$ & $136 \mathrm{ab}$ \\
\hline $\mathrm{NP}-10^{\frac{3 /}{}}$ & $154 \mathrm{a}^{\mathrm{ns}}$ & $101 \mathrm{a}$ & $107 \mathrm{ab}$ \\
\hline Dash $\mathrm{HC}^{-3 /}$ & $155 \mathrm{a}^{\mathrm{ns}}$ & $117 \mathrm{a}$ & $140 \mathrm{ab}$ \\
\hline Emultec $\mathrm{R}^{3 /}$ & $127 \mathrm{a}^{\mathrm{ns}}$ & $118 \mathrm{a}$ & $145 \mathrm{a}$ \\
\hline
\end{tabular}

1/ Testemunha com aplicação de água. 르 Testemunha aplicada somente com glyphosate. ${ }^{3 /}$ Todos os adjuvantes foram aplicados juntamente com glyphosate. ${ }^{4 /}$ Médias seguidas por letras minúsculas iguais na coluna e letras maiúsculas iguais na linha não diferem entre si pelo teste de Tukey a $5 \%$ de probabilidade. ${ }^{5 /}$ Não significativo.

Planta Daninha, Viçosa-MG, v. 33, n. 2, p. 375-386, 2015 
autores, os valores médios de amplitude relativa (variação do tamanho da gota) são altos quando se consideram pontas com indução de ar, o que não é desejável, pois há maior risco de perdas por deriva dessas gotas com menor DMV. Isso pode ser explicado, ainda, pela avaliação da correlação de Pearson entre a distância percorrida pela deriva com a densidade de gotas e o diâmetro mediano volumétrico (Figura 2). Ao se aumentar a distância de deriva, há diminuição do DMV e aumento da densidade dessas gotas de menor diâmetro.

Como se esperava, os maiores niveis de fitotoxicidade foram observados ao se utilizar a ponta XR 110.15 nas distâncias avaliadas, a qual diferiu estatisticamente das demais pontas de pulverização (Tabela 8), com exceção dos primeiros $2 \mathrm{~m}$ após a faixa de aplicação, sendo que esta não diferiu da AIXR 110.015.

Ao se considerar a fitotoxicidade nas distâncias que a esteira de aplicação percorreu para fora da área-alvo, observou-se que, para a ponta XR, não houve diferença significativa até os $4 \mathrm{~m}$ de distância do local de aplicação. Para a ponta AIXR, a maior fitotoxicidade ocorre nos primeiros $2 \mathrm{~m}$, e foi observada

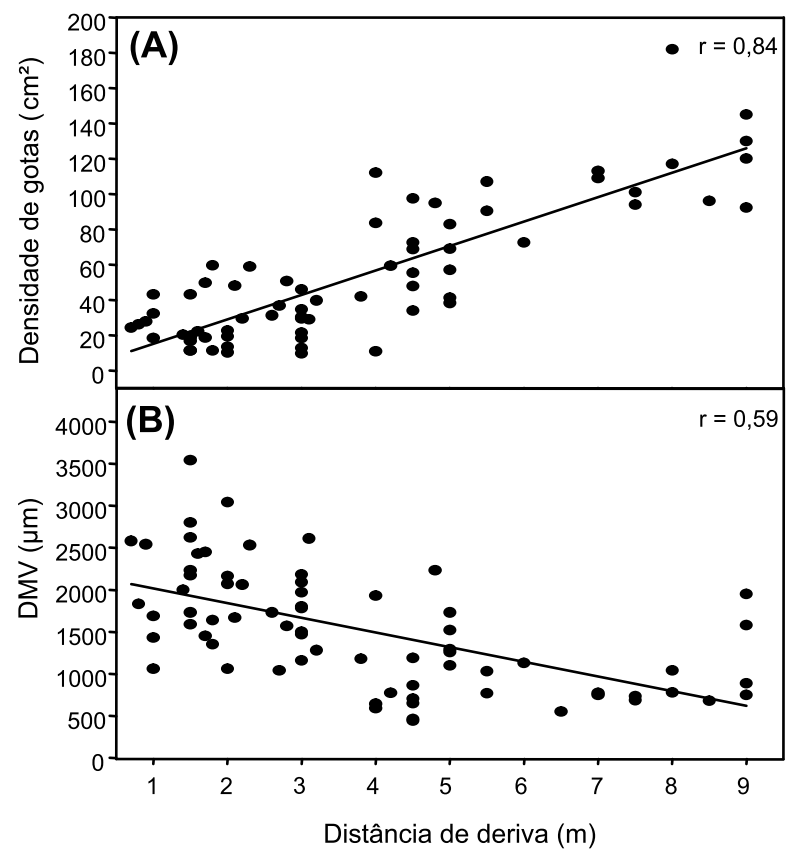

Figura 2 - Correlação de Pearson entre a distância de deriva e a densidade de gotas na faixa de aplicação (A) e o diâmetro mediano volumétrico na faixa de aplicação (B).
Tabela 8 - Fitotoxicidade observada em plantas de arroz irrigado aos 21 dias após a aplicação dos tratamentos por via terrestre

\begin{tabular}{|c|c|c|c|}
\hline \multirow{2}{*}{ Distância (m) } & \multicolumn{3}{|c|}{ Fitotoxicidade (\%) } \\
\cline { 2 - 4 } & XR 110.015 & AIXR 110.015 & TTI 110.015 \\
\hline 1 & $89^{\frac{1}{}} \mathrm{~A}^{\frac{2}{\mathrm{n}} \mathrm{3} /} /$ & $88 \mathrm{a}$ & $88 \mathrm{a}$ \\
\hline 2 & $88 \mathrm{a} \mathrm{A}$ & $72 \mathrm{a} \mathrm{A}$ & $37 \mathrm{~b} \mathrm{~B}$ \\
\hline 3 & $84 \mathrm{a} \mathrm{A}$ & $55 \mathrm{~b} \mathrm{~B}$ & $15 \mathrm{c} \mathrm{C}$ \\
\hline 4 & $73 \mathrm{a} \mathrm{A}$ & $28 \mathrm{c} \mathrm{B}$ & $0 \mathrm{~d} \mathrm{C}$ \\
\hline 5 & $53 \mathrm{~b} \mathrm{~A}$ & $14 \mathrm{c} \mathrm{B}$ & $0 \mathrm{~d} \mathrm{C}$ \\
\hline 6 & $38 \mathrm{c} \mathrm{A}$ & $0 \mathrm{~d} \mathrm{~B}$ & $0 \mathrm{~d} \mathrm{~B}$ \\
\hline 7 & $26 \mathrm{~cd} \mathrm{~A}$ & $0 \mathrm{~d} \mathrm{~B}$ & $0 \mathrm{~d} \mathrm{~B}$ \\
\hline 8 & $15 \mathrm{~d} \mathrm{~A}$ & $0 \mathrm{~d} \mathrm{~B}$ & $0 \mathrm{~d} \mathrm{~B}$ \\
\hline 9 & $9 \mathrm{~d} \mathrm{~A}$ & $0 \mathrm{~d} \mathrm{~B}$ & $0 \mathrm{~d} \mathrm{~B}$ \\
\hline
\end{tabular}

1/ Valores médios obtidos pelos tratamentos de adjuvantes com glyphosate. ${ }^{2 /}$ Médias seguidas por letras minúsculas iguais na coluna e letras maiúsculas iguais na linha não diferem entre si pelo teste de Tukey a $5 \%$ de probabilidade. ${ }^{3 /}$ Não significativo.

4/ Dados transformados por $Y t=\operatorname{arcseno} \sqrt{(y=0,5) / 100}$.

fitotoxicidade visual até os $5 \mathrm{~m}$. Já para a ponta TTI, o maior sintoma é observado $1 \mathrm{~m}$ após a faixa de aplicação e observam-se sintomas até os $3 \mathrm{~m}$ depois da faixa aplicada. Essas diferenças estão de acordo com o que foi discutido anteriormente, pois o espectro de gotas de cada ponta é diferente, mesmo quando se consideram pontas com indução de ar.

Quanto à fitotoxicidade, ao considerar distância e adjuvantes, observa-se a mesma tendência, ou seja, ao se aumentar a distância percorrida pela esteira de aplicação, diminui a fitotoxicidade, independentemente do adjuvante utilizado, o que está de acordo com os trabalhos realizados em túnel de vento por Lund (2000). Esse autor, ao avaliar deriva com diferentes pontas de pulverização e adjuvantes na calda aspergida, concluiu que o aumento da distância de coleta em relação à ponta de pulverização resulta em menor porcentagem de coleta da deriva. Contudo, ao se adicionar Emultec $\mathrm{R}^{\circledR}$ à calda de aplicação, na última distância avaliada, ainda se observa efeito da toxicidade nas plantas de arroz (Tabela 9). Considerando a utilização dos adjuvantes em cada distância, observa-se que nos primeiros $7 \mathrm{~m}$ de avaliação não houve diferença significativa na fitotoxicidade e que, aos 8 e $9 \mathrm{~m}$ de avaliação da deriva, a utilização do adjuvante Emultec $R^{\circledR}$ ocasionou maior 
Tabela 9 - Fitotoxicidade dos adjuvantes em calda com glyphosate observada em plantas de arroz irrigado aos 21 dias após a aplicação dos tratamentos por via terrestre com equipamento pressurizado por $\mathrm{CO}_{2}$

\begin{tabular}{|c|c|c|c|c|c|c|c|c|c|}
\hline \multirow{3}{*}{ Adjuvantes } & \multicolumn{9}{|c|}{ Fitotoxicidade $(\%)^{\frac{5}{}}$} \\
\hline & \multicolumn{9}{|c|}{ Distância (m) } \\
\hline & 1 & 2 & 3 & 4 & 5 & 6 & 7 & 8 & 9 \\
\hline 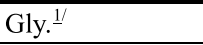 & $98^{\mathrm{ns}-3 /} \mathrm{A}^{4 /}$ & $50^{\mathrm{ns}} \mathrm{B}$ & $45^{\mathrm{ns}} \mathrm{B}$ & $28{ }^{\mathrm{ns}} \mathrm{BC}$ & $0^{\mathrm{ns}} \mathrm{C}$ & $0{ }^{\mathrm{ns}} \mathrm{C}$ & $0^{\mathrm{ns}} \mathrm{C}$ & $0 \mathrm{~b} \mathrm{C}$ & $0 \mathrm{bC}$ \\
\hline Break Thru $^{2 /}$ & $99 \mathrm{~A}$ & $77 \mathrm{AB}$ & $74 \mathrm{AB}$ & $49 \mathrm{~B}$ & $30 \mathrm{~B}$ & $14 \mathrm{C}$ & $0 \mathrm{C}$ & $0 \mathrm{~b} \mathrm{C}$ & $0 \mathrm{bC}$ \\
\hline Grap'Oil $^{2 /}$ & $100 \mathrm{~A}$ & $73 \mathrm{~A}$ & $49 \mathrm{AB}$ & $42 \mathrm{~B}$ & $32 \mathrm{BC}$ & $22 \mathrm{BC}$ & $20 \mathrm{BC}$ & $10 \mathrm{bC}$ & $0 \mathrm{bC}$ \\
\hline Grap Super Gun ${ }^{2 /}$ & $99 \mathrm{~A}$ & $63 \mathrm{AB}$ & $50 \mathrm{AB}$ & $31 \mathrm{~B}$ & $18 \mathrm{BC}$ & $18 \mathrm{BC}$ & $17 \mathrm{BC}$ & $0 \mathrm{bCC}$ & $0 \mathrm{bC}$ \\
\hline Iharol $^{2 /}$ & $98 \mathrm{~A}$ & $85 \mathrm{~A}$ & $61 \mathrm{AB}$ & $44 \mathrm{~B}$ & $33 \mathrm{BC}$ & $10 \mathrm{C}$ & $0 \mathrm{C}$ & $0 \mathrm{~b} \mathrm{C}$ & $0 \mathrm{bC}$ \\
\hline $\mathrm{NP}-10^{2 /}$ & $100 \mathrm{~A}$ & $87 \mathrm{~A}$ & $68 \mathrm{AB}$ & $29 \mathrm{BC}$ & $24 \mathrm{BC}$ & $19 \mathrm{BC}$ & $18 \mathrm{BC}$ & $4 \mathrm{bC}$ & $0 \mathrm{bC}$ \\
\hline Dash $\mathrm{HC}^{2} /$ & $100 \mathrm{~A}$ & $55 \mathrm{AB}$ & $44 \mathrm{~B}$ & $43 \mathrm{~B}$ & $41 \mathrm{~B}$ & $10 \mathrm{C}$ & $0 \mathrm{C}$ & $0 \mathrm{~b} \mathrm{C}$ & $0 \mathrm{bC}$ \\
\hline Emultec $\mathrm{R}^{2 /}$ & $100 \mathrm{~A}$ & $99 \mathrm{~A}$ & $72 \mathrm{~A}$ & $45 \mathrm{AB}$ & $24 \mathrm{~B}$ & $20 \mathrm{~B}$ & $20 \mathrm{~B}$ & $19 \mathrm{a} \mathrm{B}$ & $18 \mathrm{a} \mathrm{B}$ \\
\hline
\end{tabular}

1/ Testemunha aplicada somente com glyphosate. $\underline{2}^{/}$Todos os adjuvantes foram aplicados juntamente com glyphosate..$^{3 /}$ Não significativo.

4/ Médias seguidas por letras minúsculas iguais na coluna e letras maiúsculas iguais na linha não diferem entre si pelo teste de Tukey a 5\% de probabilidade. ${ }^{5 /}$ Dados transformados por $Y t=\operatorname{arcseno} \sqrt{(y=0,5) / 100}$.

fitotoxicidade, diferindo estatisticamente dos demais tratamentos.

Assim, a partir dos resultados deste trabalho, é possivel concluir que a redução da deriva de glyphosate está relacionada com a seleção correta da ponta de pulverização.

As pontas de pulverização TTI 110.015 e AIXR 110.015 proporcionaram menor deriva que a ponta XR 110.015, bem como menor densidade de gotas e maior diâmetro mediano volumétrico.

A utilização dos adjuvantes NP- $10^{\circledR}$ e Emultec $\mathrm{R}^{\circledR}$ com a ponta de pulverização XR 110.015 aumentou a deriva, em comparação com a aplicação de glyphosate sem adjuvantes, porém eles não diferiram dos demais aqui estudados.

$\mathrm{O}$ adjuvante Emultec $\mathrm{R}^{\circledR}$ aumentou o DMV das gotas, quando geradas pela ponta XR 110.015.

O uso dos adjuvantes Grap' Oil ${ }^{\circledR}$, Grap Super Gun ${ }^{\circledR}$, Iharol ${ }^{\circledR}$ e Dash $\mathrm{HC}^{\circledR}$ diminuiu a densidade de gotas derivadas, quando geradas pela ponta XR 110.015.

\section{AGRADECIMENTOS}

À Universidade Federal de Pelotas, pela disponibilidade de sua estrutura e viabilização das pesquisas realizadas. Ao Edital MCT/ CNPq/MEC/CAPES/CT-AGRO/CT-HIDRO/ FAPS/EMBRAPA No 22/2010 - Fundação de
Amparo à Pesquisa e Inovação do Estado de Santa Catarina (Fapesc) - contrato 6946/20119 e Conselho Nacional de Desenvolvimento Científico e Tecnológico (CNPq) - contrato 562451/2010-2. À FAPERGS - Edital Pesquisador Gaúcho, N. Proc. 12/1830-6. À CAPES, pelo auxílio financeiro.

\section{LITERATURA CITADA}

ANTUNIASSI, U. R. et al. Spray drift from aerial application on sugarcane. In: INTERNATIONAL CONFERENCE ON AGRICULTURAL ENGINEERING CIGR-AgEng 2012, Valência. Agric. Eng. Healthier Life, v. 1, n. 1, p. 1-6, 2012.

ARAÚJO, E. C.; ARAÚJO, R. M. Análise de deposição de gotas por digitalização de imagens. Pelotas: Agronautas, 2012. 16 p. (Boletim Técnico).

CHECHETTO, R. G.; ANTUNIASSI, U. R. Espectro de gotas gerado por diferentes adjuvantes e pontas de pulverização. Eng. Agric., v. 27, n. 3, p. 130-142, 2012.

COSTA, A. G. F. et al. Efeito da intensidade do vento, da pressão e de pontas de pulverização na deriva de aplicações de herbicidas em pré-emergência. Planta Daninha, v. 25, n. 1, p. 203-210, 2007.

COSTA, D. I. Eficiência e qualidade de aplicações de fungicidas, por vias terrestre e aérea, no controle de doenças foliares e no rendimento de grãos de soja e milho. 2009. 126 f. Tese (Doutorado em Agronomia) Universidade de Passo Fundo, Passo Fundo, 2009.

COUNCE, P.; KEISLING, T. C.; MITCHELL, A. J. A uniform, objective, and the adaptive system for expressing rice development. Crop Sci., v. 40, n. 2, p. 436-443, 2000. 
CUNHA, J. P. A. R. et al. Avaliação de estratégias para redução da deriva de agrotóxicos em pulverizações hidráulicas Planta Daninha, v. 21, n. 2, p. 325-332, 2003.

CUNHA, J. P. A.; TEIXEIRA, M. M.; FERNANDES, H. C. Avaliação do espectro de gotas de pontas de pulverização hidráulicas utilizando a técnica da difração do raio laser. Eng. Agríc., v. 27, p. 10-15, 2007. (Número Especial)

DERKSEN, R. C. et al. Droplet spectra and avaluation of venture and pre-orifice nozzles. Trans. ASABE, v. 42, n. 6, p. 1573-1580, 1999.

DE RUITER, H. Developments in adjuvant use for agrochemicals. Meded Rijksuniv Gent Fak Landbouwkd Toegep Biol Wet, v. 67, n. 2, p. 19-25, 2002.

FIETSAM, J. W.; YOUNG, B. G.; STEFFEN, R. W. Herbicide drift reduction to drift control agents with glyphosate. Trans. ASABE, v. 47, n. 5, p. 1405-1411, 2004.

GAZZIERO, D. L. P.; VELINI, E. D.; OSIPE, R. Procedimentos para instalação, avaliação e análise de experimentos com herbicidas. Londrina: Sociedade Brasileira da Ciência de Plantas Daninhas, 1995. 42 p.

GULART, C. A. et al. Espectro de gotas de pulverização e controle de doenças em duas cultivares de trigo. Ci. Rural, v. 43, n. 10, p. 1747-1753, 2013.

HAZEN, J. L. Adjuvants: Terminology, classification, e chemistry. Weed Technol., v. 14, n. 4, p. 773-784, 2000.

KIRK, I. W. et al. Aerial spray deposition in cotton. Trans. ASAE, v. 35, n. 5, p. 1393-1399, 1992.

LUND, I. Nozzles for drift reduction. Aspects Appl. Biol., v. 57, n. 3 , p. $97-102,2000$.

MATTHEWS, G. A. Application of pesticides to crops. London: Imperial College Press, 2000. 325 p.

MILLER, P. C. H. Spray drift and its measurement. In: MATTHEWS, G. A.; HISLOP, E. C. Application technology for crop protection. Cambridge: $\mathrm{CAB}$ International, 1993. p. 101-122.

MILLER, P. C. H.; BUTLER ELLIS, M. C. Effects of formulation on spray nozzle performance for applications from ground-based boom sprayers. Crop Protec., v. 19, n. 8 , p. $609-615,2000$.

MILLER, P. C. H. Reducing the risk of drift from boom sprayers. In: RAETANO, C. G.; ANTUNIASSI, U. R. Qualidade em tecnologia de aplicação. Botucatu: Fepaf, 2004. p. 110-124.

Planta Daninha, Viçosa-MG, v. 33, n. 2, p. 375-386, 2015
MOREIRA JR., O. Construção e validação de um túnel de vento para ensaios de estimativa da deriva em pulverizações agrícolas. 2009. 79 f. Tese (Doutorado em Agronomia) - Universidade Estadual Paulista "Júlio de Mesquita Filho", Botucatu,SP, 2009.

NUYTTENS, D. et al. Experimental study of factors influencing the risk of drift from field sprayes, Part 1: Meteorological condtions. Aspect Appl. Biol., v. 77, n. 2, p. 321-329, 2006.

OLIVEIRA, R. B.; ANTUNIASSI, U. Caracterização física e química e potencial de deriva de caldas contendo surfatantes em pulverizações agrícolas. Energia Agric., v. 27, n. 1, p. 138-149, 2012.

OZMERI, A.; CILINGIR, I. Use of colorimetric technique in determining surface coverage in spraying. Agric. Mechan. Asia, Africa Latin America, v. 23, n. 1, p. 37-38, 1992.

PIGGOTT, S.; MATTHEWS, G. A. Air induction nozzles: a solution to spray drift? Intern. Pest Control, v. 41, p. 24-28, 1999.

SANDERSON, R. et al. Relative drift potential and droplet size spectra of aerially applied propanil formulations.

Crop Protec., v. 16, n. 8, p. 717-721, 1997.

SANTOS, R. O. Níveis de deposição de produtos líquidos com aplicação aérea utilizando adjuvantes. $2007.83 \mathrm{f}$. Dissertação (Mestrado em Engenharia Agrícola) Universidade Federal de Lavras, Lavras, 2007.

SCHRÖDER, E. P. Avaliação de sistemas aeroagrícolas visando a minimização de contaminação ambiental. 2003. 66 f. Tese (Doutorado em Ciências) - Faculdade de Agronomia Eliseu Maciel, Universidade Federal de Pelotas, Pelotas, 2003.

SCHRÖDER, E. P.; LOECK, A. E. Avaliação do sistema de pulverização eletrostática aérea na redução do volume de calda e dosagem do herbicida glifosate. R. Bras. Agroci., v. 12, n. 3 , p. 319-323, 2006.

SIDAHMED, M. M. Analytical comparison of force and energy balance methods for characterizing sprays from hydraulic nozzles. Trans. ASAE, v. 41, n. 3, p. 531-536, 1998.

SOCIEDADE SUL-BRASILEIRA DE ARROZ IRRIGADO - SOSBAI. Recomendações técnicas da pesquisa para o sul do Brasil. Gravatal: 2012. 176 p.

STOCK, D.; BRIGGS, G. Physiochemical properties of adjuvants: values and applications. Weed Technol., v. 14, n. 4, p. 798-806, 2000.

VIANA, R. G. et al. Deposição de gotas no dossel da soja por diferentes pontas de pulverização hidráulica e pressões de trabalho. Eng. Agric., v. 16, n. 4, p. 428-435, 2008. 